\title{
RelB, a good prognosis predictor, links cell-cycle and migration to glioma tumorigenesis
}

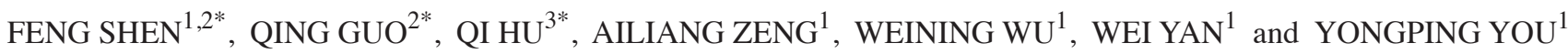 \\ ${ }^{1}$ Department of Neurosurgery, The First Affiliated Hospital of Nanjing Medical University, Nanjing, Jiangsu 210029; \\ ${ }^{2}$ Department of Oncology, People's Hospital of Taizhou, Taizhou, Jiangsu 225300; ${ }^{3}$ Department of Neurosurgery, \\ The First People's Hospital of Yueyang, Yueyang, Hunan 414000, P.R. China
}

Received April 3, 2017; Accepted January 16, 2018

DOI: $10.3892 / \mathrm{ol} .2018 .7894$

\begin{abstract}
Nuclear factor $\kappa \mathrm{B}(\mathrm{NF}-\kappa \mathrm{B})$ ex hibits an important role in inflammation and tumorigenesis. The key regulatory protein of the pathway, RELB Proto-Oncogene, NF-KB Subunit (relB), is overexpressed and associated with the pathogenesis of a variety of malignant tumors. However, the molecular features and clinical signature of relB expression in gliomas remains to be elucidated. The present study obtained the raw sequencing data of 325 glioma samples of all grades from the Chinese Glioma Genome Atlas (CGGA) database and human glioma cell line (LN229) from the Chinese Academy of Sciences cell bank. Cell proliferation, invasion and wound healing assays were used for functional annotation of relB. Western blot analysis was used for validating the protein expression of relB, matrix metalloproteinase (MMP)-2 and MMP-9 in a further 77 glioma samples. In Diffuse Glioma data, relB expression was associated with glioma grade, demonstrated a mesenchymal subtype preference and cell development association. The downregulation of relB expression inhibited glioma cell migration and invasion by regulating the MMPs in vitro. relB expression was independently associated with grade and prognosis of grade III and grade IV gliomas, suggesting that relB is a novel biomarker with therapeutic potential for predicting prognosis in glioma.
\end{abstract}

\section{Introduction}

High-grade gliomas (HGGs), including glioblastoma (GBM, World Health Organization, WHO, grade IV) and anaplastic glioma (WHO grade III), are the most frequently primary

Correspondence to: Dr Yongping You, Department of Neurosurgery, The First Affiliated Hospital of Nanjing Medical University, Guangzhou Road 300, Nanjing, Jiangsu 210029, P.R. China

E-mail: yyp19@njmu.edu.cn

*Contributed equally

Key words: relB, cell development, migration, survival, glioma brain tumors (1). Current therapeutic approaches include surgery, chemotherapy, radiotherapy, and molecular targeted therapy, however, the overall prognosis of the disease remains poor $(2,3)$. Therefore, it is critical for developing novel and effective molecular makers to assist early diagnosis and accurate prediction of prognosis in patients with glioma.

Microarray and sequencing techniques have provided better approaches for screening effective prognostic markers for human cancers $(4,5)$. It is well known that nuclear factor $\kappa B$ $(\mathrm{NF}-\kappa \mathrm{B})$ plays a significant role in inflammatory reaction and tumorigenesis (6-8). Recently, their key regulatory protein, relB, has been reported to overexpressed and associated with pathogenesis of malignancies (9). However, the molecular features and clinical signature of gliomas with relB expression remain poorly understood.

In this study, we evaluated the relB expression in the RNA sequencing of Diffuse Gliomas data and found the expression of relB was associated with glioma grade and showed a mesenchymal subtype preference. In vitro experiments demonstrated that relB reduction inhibited glioma cell migration and invasion by regulating MMPs, MMP2 and MMP9 especially $(10,11)$. Further more, some researchers find that $\mathrm{relB} / \mathrm{NF}-\kappa \mathrm{B}$ links cell cycle transition and proliferation to tumorigenesis (12-14). These data demonstrate that relB drives malignant behavior of gliomas, and it may be a novel prognostic biomarker in glioma.

\section{Materials and methods}

Human glioma tissues and cell lines. The raw sequencing data for 325 gliomas was downloaded from the Chinese Glioma Genome Atlas (CGGA) data portal (http://www.cgga.org.cn/portal.php). Human LN229 glioma cell line was obtained from the Chinese Academy of Sciences cell bank. Glioma cell line was cultured in Dulbecco's modified Eagle's medium (DMEM) (Thermo Fisher Scientific, Inc., Waltham, MA, USA), which was supplemented with $10 \%$ fetal bovine serum (Thermo Fisher Scientific, Inc.), 100 units/ml penicillin, and $100 \mathrm{ng} / \mathrm{ml}$ streptomycin (Abcam, Shanghai, China). All cells were incubated at $37^{\circ} \mathrm{C}$ in an atmosphere of $5 \% \mathrm{CO}_{2}$.

Oligonucleotides and cell transfection. The relB-siRNA, MMP9-siRNA, and MMP2-siRNA oligonucleotides were 
designed and synthesized by GenePharma and Gene chem (Shanghai, China). An siRNA that was unrelated to any human sequence was used as a negative control (NC). The plasmid containing the ORF of relB, was generated from Abcam, Shanghai, China. Blank vector was used as an NC. Then transfection complexes were formed from oligonucleotides using Lipofectamine 2000 (Thermo Fisher Scientific, Inc., Waltham, MA, USA). Transfection complexes have been added to glioma cells and incubated for 6-8 $\mathrm{h}$ before the medium was changed.

Cell proliferation assay. Cells in the logarithmic phase of growth were seeded at 3,000 per well in 96-well plates and cultured. Cell proliferation was assayed at the indicated time points using a CCK8 kit (Beyotime Institute of Biotechnology, Haimen, China) according to the manufacturer's instructions.

Invasion assay. Cell invasion assays were performed using transwell membranes coated with Matrigel (BD Biosciences, Franklin Lakes, NJ, USA). Transfected cells were plated at a density of $3 \times 10^{4}$ cells per well in the upper chamber and in serum-free medium. The lower chamber was filled with $20 \%$ FBS as a chemo-attractant. After $24 \mathrm{~h}$ of incubation, cells remaining in the upper chamber of each well were carefully removed with cotton swabs, and invading cells were fixed with $3 \%$ paraformaldehyde (Abcam, Shanghai, China), stained with crystal violet (Abcam, Shanghai, China), and counted from three independent fields (x100 magnification).

Wound healing assay. Cells were cultured until reached $90 \%$ confluence in 6-well plates. Cell layers were scratched using a 20- $\mu$ l tip to form wound gaps, washed twice with PBS and cultured. The wound healing was photographed at different time points and wounded gaps were analyzed by measuring the distance of migrating cells for three different areas for each wound.

Western blot analysis. Equal amounts of protein per lane were separated by $8 \%$ SDS-polyacrylamide gel and transferred to Polyvinylidene difluoride (PVDF) membrane. The membrane was blocked in 5\% skim milk for $2 \mathrm{~h}$ and then incubated with diluted primary antibody in 5\% w/v BSA, 1X TBS, $0.1 \%$ Tween 20 at $4^{\circ} \mathrm{C}$ with gentle shaking, overnight. The antibodies used in this study were: relB $(1: 1,000 ; 10544$, Cell Signaling Technology, Danvers, MA, USA), MMP2 (1:1,000; 87809, Cell Signaling Technology, Danvers, MA, USA), MMP9 (1:1,000; 13667, Cell Signaling Technology, Danvers, MA, USA) and cyclin A $(1: 1,000 ; 554175, \mathrm{BD}$ Pharmingen, San Diego, CA, USA). The antibody against GAPDH (glyceraldehyde-3-phosphate dehydrogenase) (Abcam, Shanghai, China) was used as a control. The specific protein was detected by using a SuperSignal protein detection kit (Abcam, Shanghai, China). The band densities of specific proteins were quantified after normalization with the density of GAPDH. Secondary antibody is HRP Goat Anti-rabbit Ig (1:1,000; 7074, Cell Signaling Technology, Danvers, MA, USA) and HRP Goat Anti-Mouse Ig (1:1,000; 554002, BD Pharmingen, San Diego, CA, USA).

Statistical analysis. Each value was obtained from at least three independent experiments and presented as means \pm SD.
Significant differences were calculated using one-way ANOVA followed by S-N-K method for three-group comparisons and t-test for two-group comparisons. The SPSS 22.0 software package was employed (SPSS, Inc., Chicago, IL, USA). Pearson correlation analysis was performed using MATLAB software (The MathWorks, Inc., Natick, Massachusetts, USA). A probability value of $<0.05$ was considered statistically significant.

\section{Results}

RelB expression was associated with glioma grade and showed a mesenchymal subtype preference. Using the RNA sequencing of Diffuse Gliomas data, we initially explored relB expression patterns in different grades of glioma tissues and found that the level of relB mRNA increased markedly in gliomas of increasing malignancy grades (Fig. 1A). Moreover, we evaluated relB expression in TCGA molecular subtypes of gliomas and found that relB showed a mesenchymal subtype preference (Fig. 1B). We further validated the protein expression level of relB in an independent group of 32 glioma patients by Immunohistochemistry (IHC) (Fig. 1C). Similar to the findings above, relB were up-regulated with ascending malignancy grades.

Low expression of relB was a better prognostic marker in anaplastic gliomas and glioblastomas. Next we investigated the correlation between relB expression and overall survival using Kaplan-Meier survival curve analysis. We found that expressing higher than mean levels of relB were associated with decreased survival relative to those with relB levels lower than the mean in anaplastic glioma and GBM patients (Fig. 2). Therefore, Low expression of relB was a better prognostic marker in anaplastic gliomas and glioblastomas.

relB-associated genes were mainly involved in cell-cycle and migration biological process. After Pearson correlation analysis of the RNA sequencing data, the significantly positively correlated genes were used for $\mathrm{GO}$ analysis. Gene set variation analysis with relB expression was analyzed by GSVA package of R.Gene list was obtained from GSVA date package (15). As illustrated in Fig. 3A and B, function annotation of relB which was performed by GO analysis and GSVA revealed its correlation with cell-cycle and migration biological process. Furthermore, With the increase of relB expression, the expression of MMP family genes increased $(\mathrm{P}<0.05)$. MMP family genes were found to positively correlate with relB expression (Fig. 4). In particular, MMP2 and MMP9 were known to promote cell invasion and migration in cancers. Overall, relB may involves in glioma invasion, migration, and cell-cycle biological process.

Repression of relB induces glioma cell cycle arrest. We then constructed a lentivirus containing a siRNA sequence targeting relB to verify whether relB plays a potentially functional role in glioma. After $48 \mathrm{~h}$ of infection, relB protein expression levels in LN229 were decreased (Fig. 5A). To investigate the biological implication of relB in glioma, we performed functional assays to determine the influence of relB on glioma cell proliferation. Decreased relB expression in LN229 inhibited the proliferation of cell line (Fig. 5B). 
A

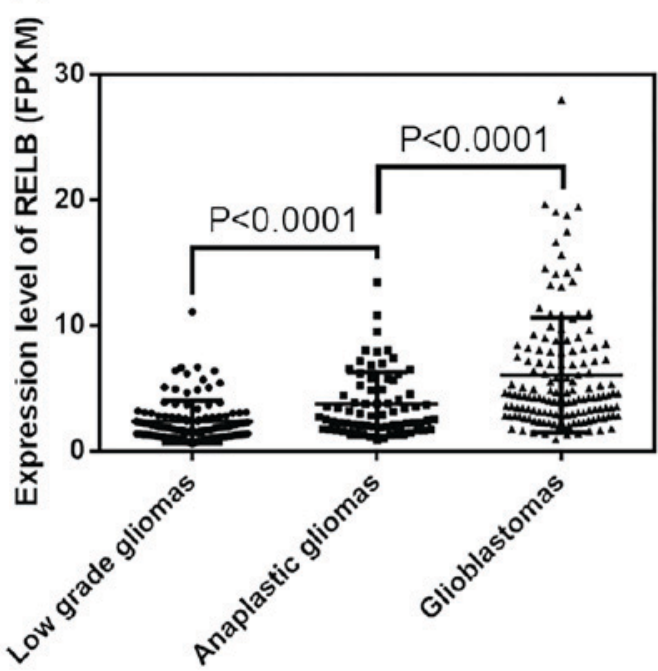

B

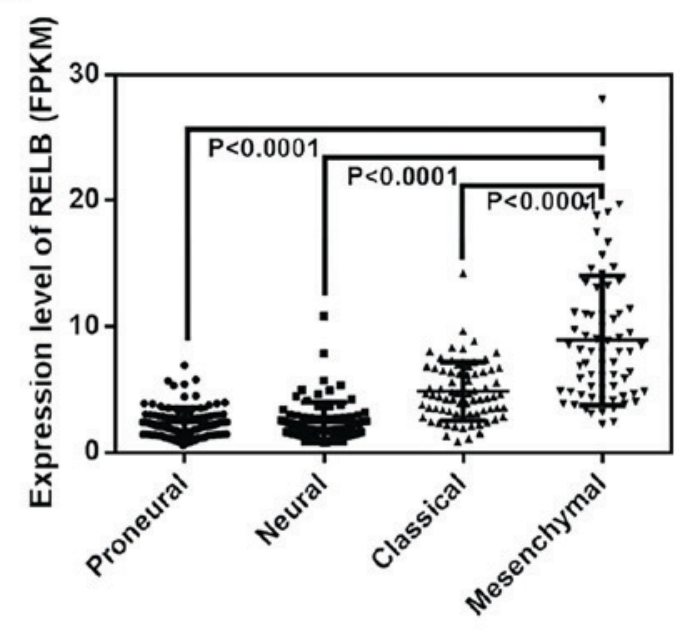



Figure 1. The expression difference of relB in different grade and pathologcal type gliomas. (A, B) A single spot was the expression value of relB of an individual patient. Lines in the middle were the mean expression value (A and B). Immunocytochemical staining of relB in different grade tumor tissues (mgnification, x200) (C) low grade gliomas, (D) anaplastic gliomas and (E) glioblastomas.
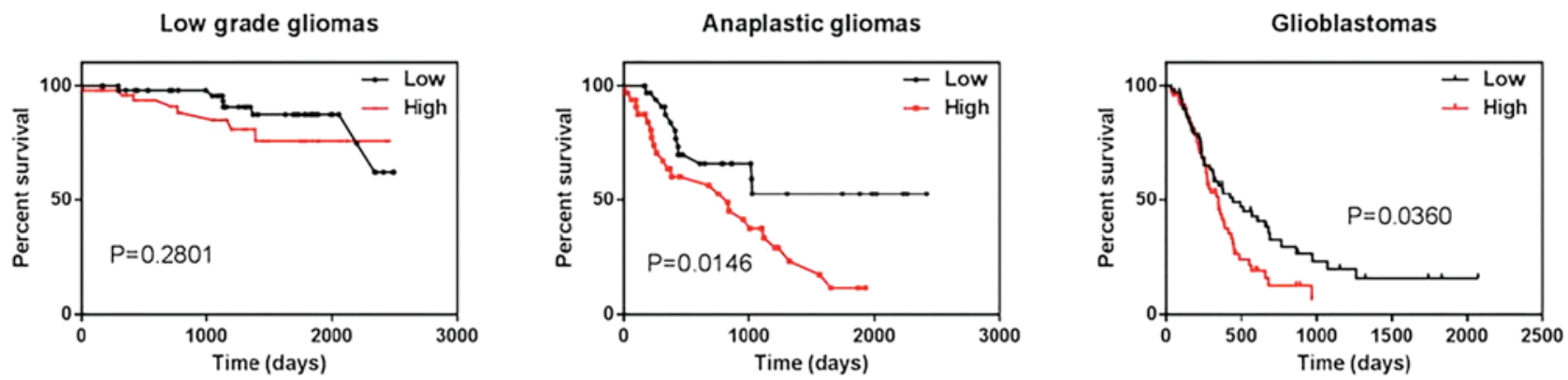

Figure 2. The prognostic value of relB in CGGA. According to relB expression level, patients with anaplastic or GBM could be divided into two groups with significantly different prognosis.

Moreover, flow cytometry analysis showed that the cell cycle was blocked in the G0-G1 phase as a result of decreased relB (Fig. 5C and D). A significant decrease of cyclin A protein expression level in the LN229 cells was observed after relB knockdown compared with the control group (Fig. 5E).

The down-regulation of relB expression inhibits glioma cell migration and invasion by regulating the MMPs in vitro. To explore the potential role of relB on the invasiveness of glioma cells, wound healing and transwell assay were employed in vitro. As shown in Fig. $6 \mathrm{~A}$ and $\mathrm{B}$, relB knockdown resulted in a marked decrease in cell invasion compared to the control group. Subsequently, the percentage of migrating cells in relB knockdown group was significantly lower than that of the control group (Fig. 6C and D). MMPs family genes were critical for cancer cell migration and invasion. Our bioinformatics analysis implied that MMP family genes were positively correlated with relB. In particular, MMP2 and MMP9 were known to promote cell invasion and migration in cancer cells (16-18). Therefore, we further tested MMP2 and MMP9 expression in relB knockdown and control cells by western blotting. Compared with the control cells, relB knockdown cells showed decreased MMP9 and MMP2 expression (Fig. 6E). Furthermore, transwell and wound healing assays showed that overexpression of relB promoted glioma cell invasion and migration whereas introduction of 
A
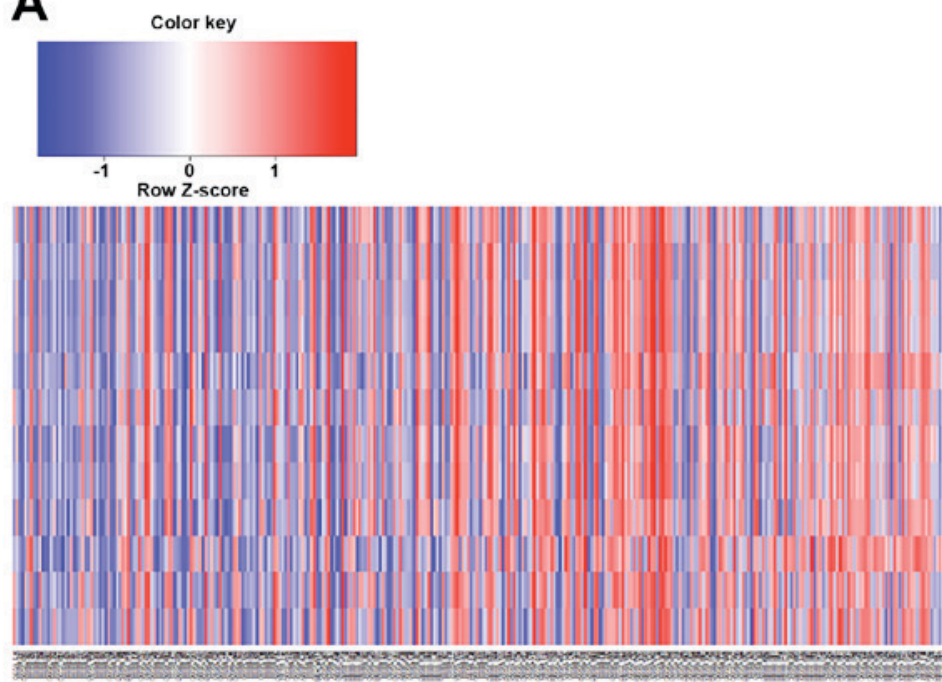

CELL_CYCLE_CHECKPOINT_GO_0000075

CELL_CYCLE_GO_0007049

CELL_CYCLE_PHASE

CELL_CYCLE_PROCESS

G1_S_TRANSITION_OF_MITOTIC_CELL_CYCLE

INTERPHASE_OF_MITOTIC_CELL_CYCLE

M_PHASE_OF_MITOTIC_CELL_CYCLE

MITOTIC_CELL_CYCLE

MITOTIC_CELL_CYCLE_CHECKPOINT

POSITIVE_REGULATION_OF_CELL_CYCLE

REGULATION_OF_CELL_CYCLE

REGULATION_OF_MITOTIC_CELL_CYCLE

B
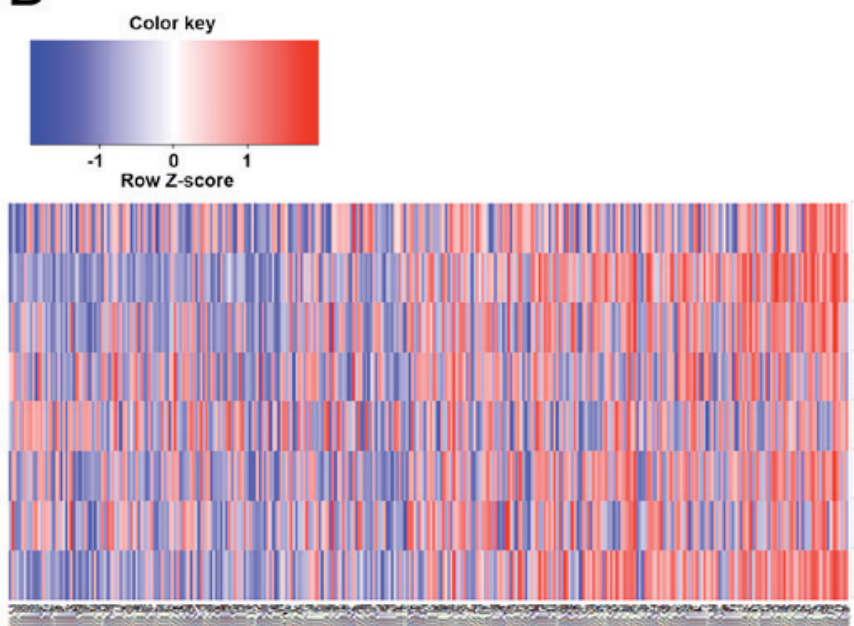

KEGG_GLYCOLYSIS_GLUCONEOGENESIS KEGG_LEUKOCYTE_TRANSENDOTHELLAL_MIGRATION REGULATION_OF_CELL_MIGRATION

POSITIVE_REGULATION_OF_CELL_MIGRATION

ENDOTHELIAL_CELL_MIGRATION

CELL_MIGRATION

NEGATIVE_REGULATION_OF_CELL_MIGRATION

LEUKOCYTE_MIGRATION

Figure 3. (A) Using GO analysis. It was showed that genes positively correlated with relB expression consist in cell-cycle process. (B) Though GO analysis. It was showed that genes positively correlated with relB expression consist in migration biological process.

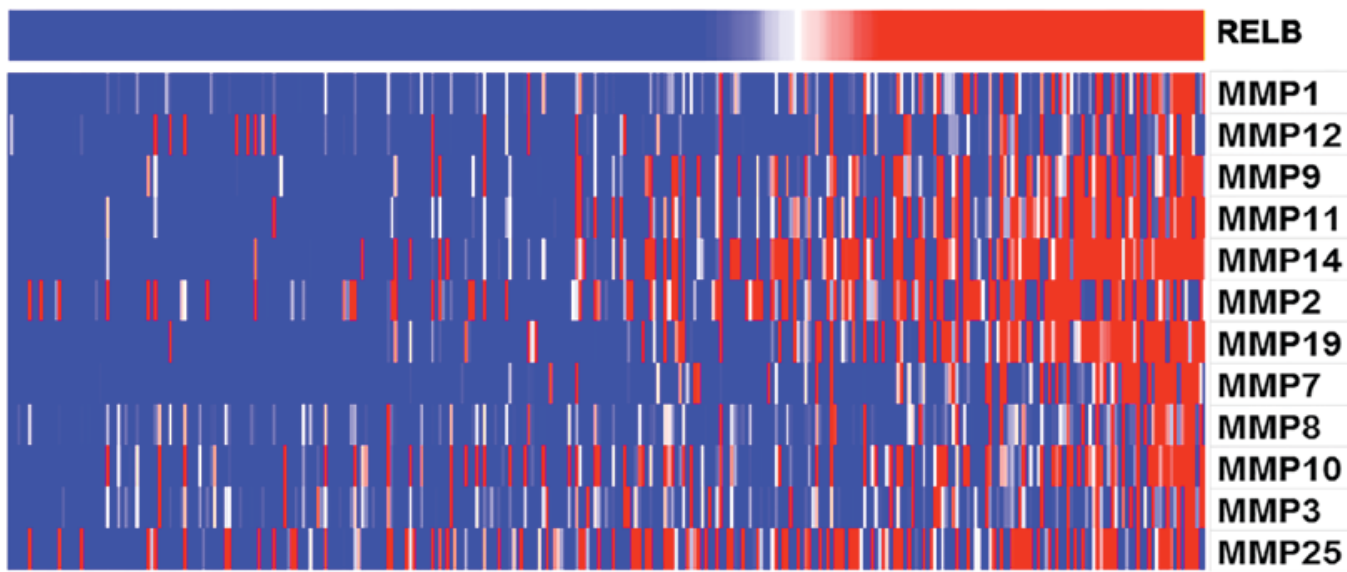

Figure 4. relB expression showed a MMPs preference. For each patient, MMPs preference were annotated and listed in the lower part, which was obtained from CGGA database. It was showed that MMPs preference positively correlated with relB expression $(\mathrm{P}<0.05)$.

si-MMP9 or si-MMP2 abrogated relB overexpression induced cellular invasion and migration. These results suggest that
relB modulates malignant progression at least partially through MMP9 and MMP2. 
A

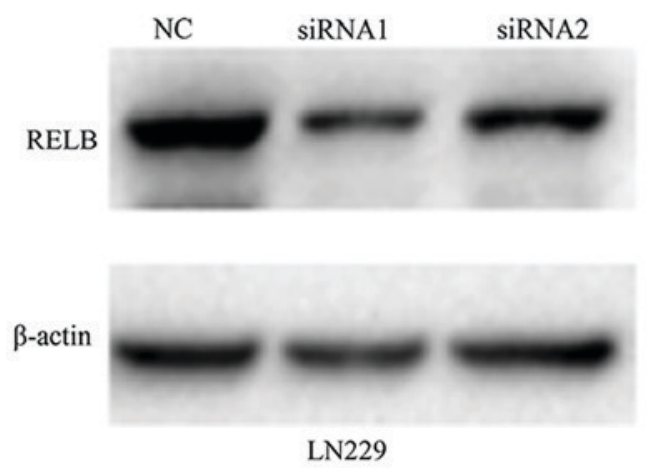

C

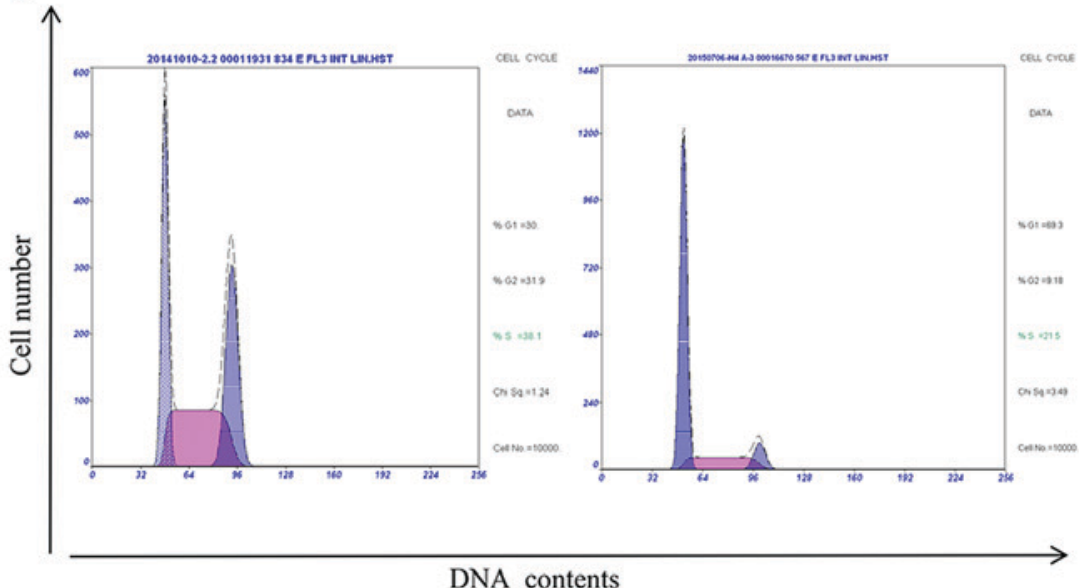

B

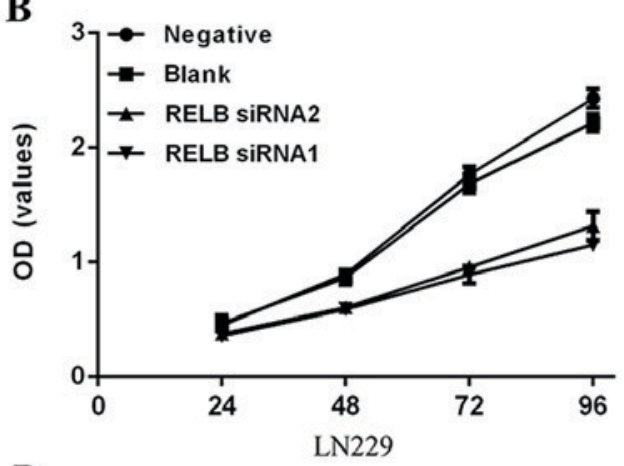

D

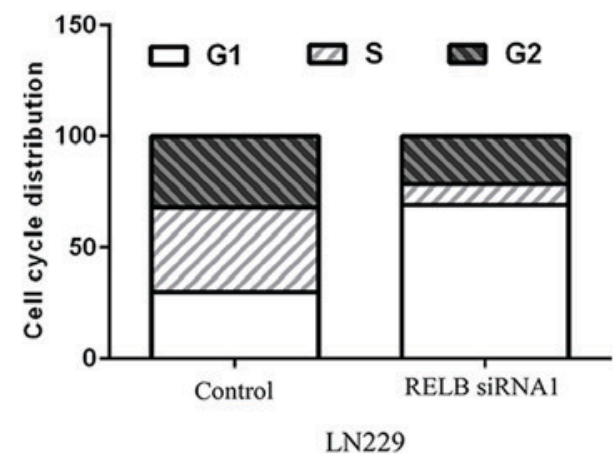

LN229

E

$\mathrm{NC}$

si-RELB

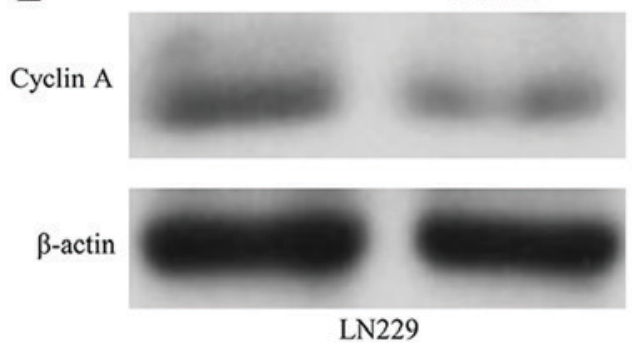

Figure 5. relB might regulates cell proliferation by influencing the cell-cycle distribution. (A) relB protein expression was detected with weston-blot after transfection with siRNA-1, siRNA-2. (B) cell viability was detected with a CCK8 assay after transfection with siRNA-1, siRNA-2 for $24,48,72$ and 96 h. (C, D) The distribution of the G0/G1, S, and G2/M phases after relB knockdown by siRNA. (E) The expression of cell-cycle associated protein Cyclin A was detected with weston-blot after relB knockdown by siRNA.

\section{Discussion}

Glioma is the most common lethal intracranial tumor in adults. Despite of tremendous efforts work on developing multimodal treatments and mechanisms, the overall prognosis of glioma remains poor (1-3). It is a matter of great urgency to identified novel biomarkers for patients of particular High-grade gliomas (HGGs).

RelB is originally found as a key transcription factors of Nuclear factor kB (NF-kB) which regulates a wide range of biological processes, such as cell survival, immune and inflammatory responses (19-21). Emerging evidences have implicated that relB plays an important role in the progression in different types of cancers. In prostate cancer, relB promotes cancer cell growth and decreases the radiosensitivity of cancer cells $(22,23)$. In breast tumor, relB enhances cellular survival and shows a highly invasive phenotypes preference (24). In chronic lymphocytic leukemia (CLL) cells, overexpression relB increases the sensitivity of CLL cells to the proteasome inhibitor, bortezomib (25). In the present study, we demonstrate that relB expression is associated with glioma grade and shows a mesenchymal subtype preference. Besides, low expression of relB is a better prognostic marker in anaplastic gliomas and glioblastomas. Therefore, our results suggest that relB may be associated with glioma progression.

To further clarify the role of relB in the development and progression of glioma, bioinformatics and experiment in vitro were applied to analyze the molecular function of relB. Through bioinformatics analysis, relB-associated genes were mainly involved in cell-cycle and migration biological process. To further verify the biological behavior of relB 

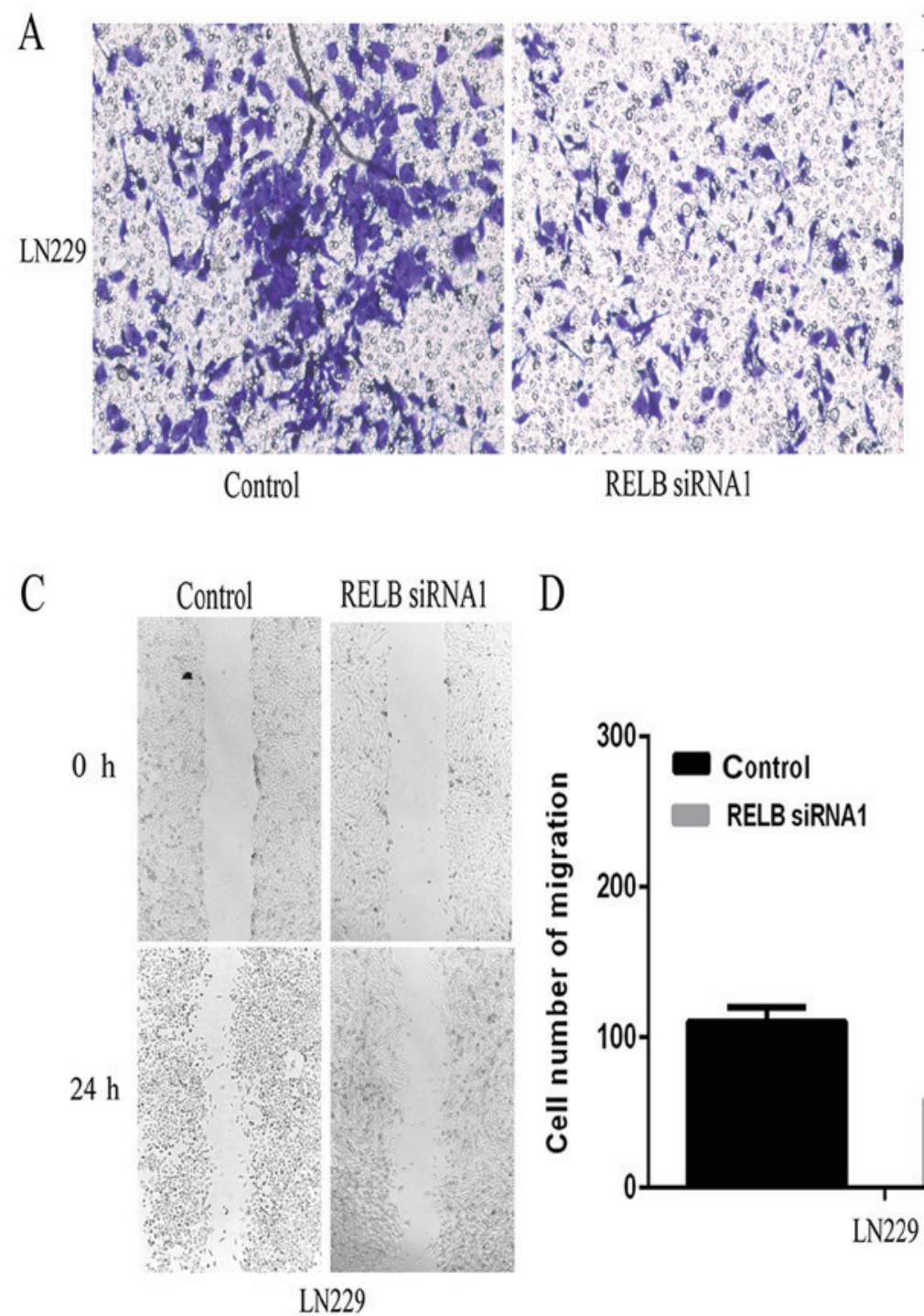

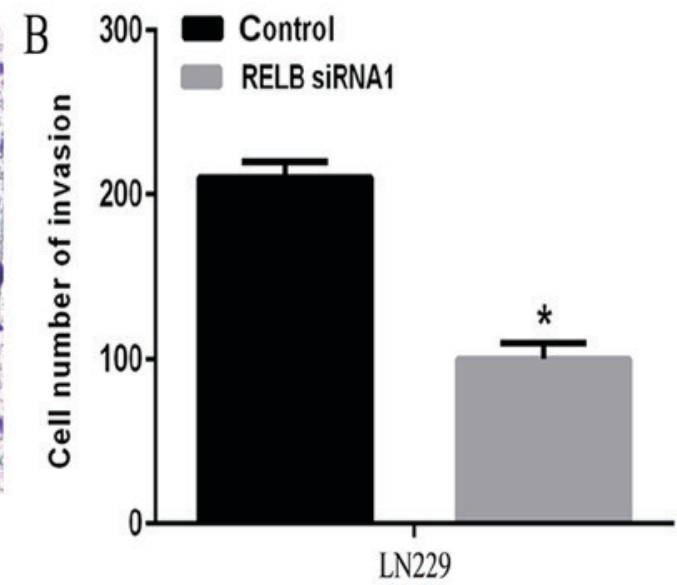

E


LN229

Figure 6. relB might influnces invasiveness of glioma cells. (A, B) Transwell assays showed that cells transfected with relB siRNA1 presented less invas-ion ability than those with relB-control $\left({ }^{*} \mathrm{P}<0.05\right)$. (C, D) Wound healing assays showed that glioma cells transfected with relB siRNA1 may close the wound more slowly when compared to those with miR-control ( $\left.{ }^{*} \mathrm{P}<0.05\right)$. (E) The level of MMP2 and MMP9 proteins were decreased in the relB siRNA group compared with relB siRNA NC group.

in gliomas, we constructed lentiviral vectors expressing nonsense control or relB siRNA, and subsequently infected the LN229 glioma cells. In vitro assay, Repression of relB induces cell cycle arrest and inhibits glioma cell migration and invasion of glioma cells. The basement membrane consist of a network of extracellular matrix (ECM) proteins which participate in tumor growth, invasion , migration and tumor angiogenesis (26). MMPs are a large family of $\mathrm{Ca}^{2+}$ and $\mathrm{Zn}^{+}$ dependent endopeptidases, especially among members MMP2 and MMP9, possessing to hydrolyze components of the basement membrane $(27,28)$. Furthermore, our results suggest that relB modulates malignant progression at least partially through MMP9 and MMP2.

In summary, our results reveal that relB is upregulated in glioma and exhibit pro-oncogenic activity partially through MMP9 and MMP2. Importantly, relB can be used as a potential diagnostic and prognostic marker for some HGGs, especially for mesenchymal subtype glioblastoma.

\section{References}

1. Wang Y and Jiang T: Understanding high grade glioma: Molecular mechanism, therapy and comprehensive management. Cancer Lett 331: 139-146, 2013.

2. van den Bent M, Chinot OL and Cairncross JG: Recent developments in the molecular characterization and treatment of oligodendroglial tumors. Neuro Oncol 5: 128-138, 2003.

3. Kalpathy-Cramer J, Gerstner ER, Emblem KE, Andronesi OC and Rosen B: Advanced magnetic resonance imaging of the physical processes in human glioblastoma. Cancer Res 74: 4622-4637, 2014.

4. Van Schaeybroeck S, Allen WL, Turkington RC and Johnston PG: Implementing prognostic and predictive biomarkers in CRC clinical trials. Nat Rev Clin Oncol 8: 222-232, 2011.

5. Reis-Filho JS and Pusztai L: Gene expression profiling in breast cancer: Classification, prognostication, and prediction. Lancet 378: 1812-1823, 2011.

6. Hayden MS and Ghosh S: NF- $\kappa$ B, the first quarter-century: Remarkable progress and outstanding questions. Genes Dev 26: 203-234, 2012.

7. Perkins ND: The diverse and complex roles of NF- $\kappa B$ subunits in cancer. Nat Rev Cancer 12: 121-132, 2012.

8. DiDonato JA, Mercurio F and Karin M: NF- $\kappa B$ and the link between inflammation and cancer. Immunol Rev 246: 379-400, 2012. 
9. Cha ST, Tan CT, Chang CC, Chu CY, Lee WJ,Lin BZ, Lin MT and Kuo ML: Retraction: G9a/RelB regulates self-renewal and function of colon-cancer-initiating cells by silencing Let-7b and activating the K-RAS/ $\beta$-catenin pathway. Nat Cell Biol 19: 76, 2016.

10. Kitamura T, Kometani K, Hashida H, Matsunaga A, Miyoshi H, Hosogi H, Aoki M, Oshima M, Hattori M, Takabayashi A, et al: SMAD4-deficient intestinal tumors recruit CCR1+ myeloid cells that promote invasion. Nat Genet 39: 467-475, 2007.

11. Kenagy RD, Hart CE, Stetler-Stevenson WG and Clowes AW: Primate smooth muscle cell migration from aortic explants is mediated by endogenous platelet-derived growth factor and basic fibroblast growth factor acting through matrix metalloproteinases 2 and 9. Circulation 96: 3555-3560, 1997.

12. Ge QL, Liu SH, Ai ZH, Tao MF, Ma L, Wen SY, Dai M, Liu F, Liu HS, Jiang RZ, et al: RelB/NF- $\kappa \mathrm{B}$ links cell cycle transition and apoptosis to endometrioid adenocarcinoma tumorigenesis. Cell Death Dis 7: e2402, 2016.

13. Sau A, Lau R, Cabrita MA, Nolan E, Crooks PA, Visvader JE and Pratt MA: Persistent activation of NF- $\mathrm{B}$ in BRCA1-deficient mammary progenitors drives aberrant proliferation and accumulation of DNA damage. Cell Stem Cell 19: 52-65, 2016

14. Gardella KA, Muro I, Fang G, Sarkar K, Mendez O and Wright CW: Aryl hydrocarbon receptor nuclear translocator (ARNT) isoforms control lymphoid cancer cell proliferation through differentially regulating tumor suppressor p53 activity. Oncotarget 7: 10710-10722, 2016.

15. Hänzelmann S, Castelo R and Guinney J: GSVA: Gene set variation analysis for microarray and RNA-seq data. BMC Bioinformatics 14: 7, 2013.

16. Zheng H, Takahashi H, Murai Y, Cui Z, Nomoto K, Niwa $H$ Tsuneyama K and Takano Y: Expressions of MMP-2, MMP-9 and VEGF are closely linked to growth, invasion, metastasis and angiogenesis of gastric carcinoma. Anticancer Res 26: 3579-3583, 2006.

17. Deryugina EI and Quigley JP: Tumor angiogenesis: MMP-mediated induction of intravasation- and metastasis-sustaining neovasculature. Matrix Biol 44-46: 94-112, 2015.

18. Gao M, Zhang X, Li D, He P, Tian W and Zeng B: Expression analysis and clinical significance of eIF4E, VEGF-C, E-cadherin and MMP-2 in colorectal adenocarcinoma. Oncotarget 7 $85502-85514,2016$

19. Guo F, Tänzer S, Busslinger M and Weih F: Lack of nuclear factor-kappa B2/p100 causes a RelB-dependent block in early B lymphopoiesis. Blood 112: 551-559, 2008.
20. Bellet MM, Zocchi L and Sassone-Corsi P: The RelB subunit of $\mathrm{NF} \kappa \mathrm{B}$ acts as a negative regulator of circadian gene expression. Cell Cycle 11: 3304-3311, 2012.

21. McMillan DH, Baglole CJ, Thatcher TH, Maggirwar S, Sime PJ and Phipps RP: Lung-targeted overexpression of the NF- $\mathrm{KB}$ member RelB inhibits cigarette smoke-induced inflammation. Am J Pathol 179: 125-133, 2011.

22. Xu Y, Josson S, Fang F, Oberley TD, St Clair DK, Wan XS, Sun Y, Bakthavatchalu V, Muthuswamy A and St Clair WH: RelB enhances prostate cancer growth: Implications for the role of the nuclear factor-kappaB alternative pathway in tumorigenicity. Cancer Res 69: 3267-3271, 2009.

23. Zhu L, Zhu B, Yang L, Zhao X, Jiang H and Ma F: RelB regulates $\mathrm{Bcl}-\mathrm{xl}$ expression and the irradiation-induced apoptosis of murine prostate cancer cells. Biomed Rep 2: 354-358, 2014.

24. Mineva ND, Wang X, Yang S, Ying H, Xiao ZX, Holick MF and Sonenshein GE: Inhibition of RelB by 1,25-dihydroxyvitamin D3 promotes sensitivity of breast cancer cells to radiation. J Cell Physiol 220: 593-599, 2009.

25. Xu J, Zhou P, Sun A and Guo F: Effect of nuclear transcription factor RelB on the proteasome inhibitor-sensitivity of chronic lymphocytic leukemia cells. Zhonghua Xue Ye Xue Za Zhi 35: 524-527, 2014 (In Chinese).

26. Roskelley CD, Srebrow A and Bissell MJ: A hierarchy of ECM-mediated signalling regulates tissue-specific gene expression. Curr Opin Cell Biol 7: 736-747, 1995.

27. Park HI, Ni J, Gerkema FE, Liu D, Belozerov VE and Sang QX: Identification and characterization of human endometase (Matrix metalloproteinase-26) from endometrial tumor. J Biol Chem 275: 20540-20544, 2000.

28. Shah FD, Shukla SN, Shah PM, Shukla HK and Patel PS: Clinical significance of matrix metalloproteinase 2 and 9 in breast cancer. Indian J Cancer 46: 194-202, 2009.

This work is licensed under a Creative Commons Attribution-NonCommercial-NoDerivatives 4.0 International (CC BY-NC-ND 4.0) License. 